\title{
Analysis on Chaotic Mechanism of Direct-Drive Permanent Magnet Synchronous Generators Based on Lyapunov Stability Theory
}

\author{
Li Yang $^{1,2}$, Tianmin Huang ${ }^{1 *}$, Lin Deng ${ }^{1,2}$, Yingfeng Zeng ${ }^{1,2}$, Sudan Huang ${ }^{3}$ \\ ${ }^{1}$ School of Electrical Engineering, Southwest Jiaotong University, Chengdu 610031, China \\ ${ }^{2}$ Electrical \& Information Engineering Department, Sichuan Engineering Technical College, Deyang 618000, China \\ ${ }^{3}$ Shenzhen Key Laboratory of Electromagnetic Control, Shenzhen University, Shenzhen 518060, China
}

Corresponding Author Email: tmhuang@home.swjtu.edu.cn

https://doi.org/10.18280/ejee.210607

Received: 10 May 2019

Accepted: 6 September 2019

\section{Keywords: \\ direct-drive permanent magnet synchronous generator (D-PMSG), chaotic features, affine transform, bifurcation, Lyapunov stability}

\begin{abstract}
The direct-drive permanent magnet synchronous generator (D-PMSG) is a highly nonlinear dynamic system. This paper aims to explore the complex chaotic motions or prominent chaotic features (e.g. the limit cycle) of the D-PMSG under certain parameter conditions and external inputs. Specifically, the mathematical model of the PMSG in d-q reference frame was converted into a dimensionless Loren chaotic equation through affine transform and timescale transform. Then, the Lyapunov stability theory was introduced to explore the eigenvalues of the Jacobian matrix corresponding to each equilibrium point under different inputs. On this basis, the author discussed how the D-PMSG shifts from equilibrium state to chaotic motions, and derived the conditions for the generation of the limit cycle. Finally, the chaotic features of the system were simulated with the aid of three-phase diagram, bifurcation map and the Lyapunov exponent (LE) spectrum. The simulation results show that the chaotic attractor will appear in the system, when system parameters change or the wind speed varies, posing a serious threat to the stable operation of the D-PMSG.
\end{abstract}

\section{INTRODUCTION}

Wind power is one of the fastest growing clean energy sources [1]. The largescale wind turbines at home and abroad either use doubly-fed asynchronous generator (DFAG) or permanent magnet synchronous generator (PMSG). Compared with the DFAG [2, 3], the PMSG [4, 5] boasts a simple structure and a high efficiency, eliminating the need for rotor excitation or drawing draw reactive power from the grid.

With the rapid development of offshore wind power, the direct-drive PMSG (D-PMSG) [6, 7] has become the new trend for wind power generation, because it adopts the directdrive mode without the gearbox, induces a limited transmission loss, requires a low maintenance cost, and achieves a high reliability. As its name suggests, the D-PMSG is obviously centering on the PMSG.

The PMSG faces chaotic phenomena like the intermittent oscillations of torque and speed and the instability of control effect, when its parameters fall within specific intervals $[8,9]$. To date, many domestic and foreign scholars have explored deep into the chaotic features of the PMSG, yielding fruitful results. For example, Hemate [10] set up a PMSG model, and analyzed the conditions for the model to produce bifurcation and chaos. Li et al. [11] introduced the Lyapunov theory to the mathematical model of the PMSG, and derived the parameter conditions for three dynamic features of the PMSG, namely, steady state, limit cycle and chaos. Based on the nonlinear electrotechnical coupling model for the PMSG, Chen et al. [12] provided the analytical criteria for different parameter conditions by the Melnikov method, and demonstrated that the PMSG may suffer from bifurcation, chaos, etc. when the system parameters fall into certain intervals; the demonstration was carried out by the Poincare map and the bifurcation theory. Xue et al. [13] analyzed the chaotic features of the fractional PMSG model, using phase diagram, bifurcation diagram and Lyapunov exponent. Chang et al. [14] designed a nonsingular fast terminal sliding mode controller with extended state observer, which effectively suppresses the chaos in PMSG chaotic systems with uncertain disturbances.

Like the PMSG, the D-PMSG also faces chaotic motions under certain parameter values or working conditions. During power generation, the chaotic motions bring about intermittent oscillations in torque, speed and output power, resulting in unstable control and irregular electromagnetic noises. In this case, the D-PMSG will have substantial oscillations and fluctuations in its output power, exerting a huge impact on the grid. Yang and Li [15] was the first to discover the complex chaotic motions in the D-PMSG, and developed a sliding mode variable structure controller that effectively curbs the chaos. Wang et al. [16] discussed the chaotic phenomenon when all the inputs are zeros, and controlled the chaotic motions only on the PMSG model. Chen et al. [17] proposed a sliding mode extreme search algorithm based on chaotic particle swarm optimization (PSO), aiming to track the maximum power point (MPP) of wind power systems. Cao et al. [18] studied the chaotic features of wind power systems under changing wind speeds. Despite the above studies, there are relatively few in-depth reports on the chaotic phenomenon of the D-PMSG and the relevant control methods. In a wind power system, the emergence of a chaotic attractor will seriously affect the stable operation and grid connection of the system.

To pinpoint the chaotic features of the D-PMSG, this paper converts the mathematical model of the system into a 
dimensionless Lorenz model through linear affine transform and timescale transform. Then, the Lyapunov theory was applied to investigate the generation of limit cycle and chaotic motions of the D-PMSG under two different inputs, which theoretically proves the existence of chaotic motions in the system under certain conditions. Through simulation, it is confirmed that, under different inputs, the D-PMSG will enter the chaotic state when some parameters are changed or the wind speed varies, posing a serious threat to the stable operation of the direct-drive wind turbine.

\section{MATHEMATICAL MODEL OF THE D-PMSG}

\subsection{Model of wind turbine}

Judging by its aerodynamics, the torque of the wind wheel can be expressed as $[19,20]$ :

$$
\begin{gathered}
T_{r}=\frac{1}{2} \rho \operatorname{Arv}^{2} C_{q}(\lambda, \beta) \\
\lambda=\frac{\omega_{r} r}{v}
\end{gathered}
$$

where, $T_{r}$ is the aerodynamic torque of the wind wheel $(N \cdot m)$; $\rho$ is air density $\left(\frac{\mathrm{kg}}{\mathrm{m}^{3}}\right) ; A=\pi r^{2}$ is the area of the wind wheel $\left(\mathrm{m}^{2}\right) ; \lambda$ is the tip-speed ratio; $\beta$ is the pitch angle $\left(^{\circ}\right) ; \omega_{r}$ is the angular velocity of the wind wheel $(\mathrm{rad} / \mathrm{s}) ; C_{q}=C_{q}(\lambda, \beta)$ is the torque coefficient, a nonlinear function of $\lambda$ and $\beta ; r$ is the radius of the wind wheel $(m) ; v$ is the wind speed $\left(\frac{m}{s}\right)$.

\subsection{Model of the PMSG}

The PMSG uses three-phase symmetrical windings. Here, the saturation eddy current at the motor core and the hysteresis loss are ignored, while the $\mathrm{d}$-axis and q-axis components $\left(i_{d}\right.$ and $i_{q}$ ) of the stator current, plus the angular velocity of the rotor $\omega_{g}$, are taken as state variables. On this basis, the surfacemounted PMSG with uniform air gaps can be modelled as [21]:

$$
\begin{aligned}
\frac{\mathrm{d} i_{d}}{d t} & =-\frac{R_{s}}{L} i_{d}+n_{p} i_{q} \omega_{g}+\frac{u_{d}}{L} \\
\frac{\mathrm{d} i_{q}}{d t} & =-\frac{R_{s}}{L} i_{q}-n_{p} i_{d} \omega_{g}-\frac{n_{p} \psi_{f}}{L} \omega_{g}+\frac{u_{q}}{L} \\
\frac{\mathrm{d} \omega_{g}}{d t} & =\frac{3 n_{p} \psi_{f}}{2 J_{e q}} i_{q}-\frac{B_{m}}{J_{e q}} \omega_{g}-\frac{T_{L}}{J_{e q}}
\end{aligned}
$$

where, $u_{x}$ and $i_{x}$ are the generator-end voltage and stator current at axis $x(x=d, q)$, respectively; $R_{s}$ is the winding resistance of the stator; $n_{p}$ is the number of pole pairs of the generator; $\omega_{g} \approx \omega_{r}$ is the angular velocity of the rotor; $J_{e q}$ is the equivalent moment of inertia of the generator; $\psi_{f}$ is the flux linkage of the permanent magnet; $B_{m}$ is the rotational viscosity of the generator; $T_{L}=T_{r}$ is the load torque.

\section{CHAOTIC FEATURES OF THE D-PMSG}

Through affine transform $x=\varsigma \tilde{x}\left(x=\left[i_{d}, i_{q}, \omega_{g}\right]^{T} ; \tilde{x}=\right.$ $\left.\left[\tilde{l}_{d}, \tilde{\imath}_{q}, \widetilde{\omega}_{g}\right]^{T}\right)$ and timescale transform $t=\tau \tilde{t}$, the Lorenz chaotic model of the D-PMSG [22] can be established as:

$$
\begin{aligned}
& \frac{d \tilde{i}_{d}}{d \tilde{t}}=-\tilde{i}_{d}+\tilde{i}_{q} \tilde{\omega}_{g}+\tilde{u}_{d} \\
& \frac{d \tilde{i_{q}}}{d \tilde{t}}=-\tilde{i}_{q}-\tilde{i}_{d} \tilde{\omega}_{g}+\gamma \tilde{\omega}_{g}+\tilde{u}_{q} \\
& \frac{d \tilde{\omega}_{g}}{d \tilde{t}}=\sigma\left(\tilde{i}_{q}-\tilde{\omega}_{g}\right)-\tilde{T}_{r}
\end{aligned}
$$

where, $\varsigma_{1}=\varsigma_{2}=\frac{B_{m}}{\tau \psi_{f} n_{p}^{2}} ; \varsigma_{3}=\frac{1}{\tau n_{p}} ; \tau=\frac{L}{R_{S}} ; \sigma=\frac{\tau B_{m}}{J_{e q}} ; \gamma=$ $\frac{-\left(3 \tau n_{p}^{2} \psi_{f}^{2}\right)}{\left(2 B_{m} L\right)} ; \tilde{u}_{d}=\frac{\tau}{\varsigma_{1} L} u_{d} ; \tilde{u}_{q}=\frac{\tau}{\varsigma_{1} L} u_{q} ; \tilde{T}_{r}=\frac{\tau}{\varsigma_{3} J_{e q}} T_{r} ; \sigma$ and $\gamma$ are free numbers determined by system parameters.

The equilibrium point of system (4) satisfies:

$$
\begin{gathered}
\tilde{i}_{d}^{e}=\left(\tilde{\omega}_{g}^{e}\right)^{2}+\frac{\tilde{T}_{L}}{\sigma} \tilde{\omega}_{g}^{e}+\tilde{u}_{d} \\
\tilde{i}_{\mathrm{q}}^{e}=\tilde{\omega}_{g}^{e}+\frac{\tilde{T}_{L}}{\sigma} \\
\left(\tilde{\omega}_{g}^{e}\right)^{3}+\frac{\tilde{T}_{L}}{\sigma}\left(\tilde{\omega}_{g}^{e}\right)^{2}+\left(\tilde{u}_{d}-\gamma+1\right) \tilde{\omega}_{g}^{e}+\frac{\tilde{T}_{L}}{\sigma}-\tilde{u}_{q}=0
\end{gathered}
$$

where, $\left(\tilde{\imath}_{d}^{e}, \tilde{l}_{q}^{e}, \widetilde{\omega}_{g}^{e}\right)$ is the equilibrium point of the system.

The Jacobian matrix corresponding to the equilibrium point can be expressed as:

$$
J=\left[\begin{array}{ccc}
-1 & \tilde{\omega}_{g}^{e} & \tilde{i}_{q}^{e} \\
-\tilde{\omega}_{g}^{e} & -1 & \gamma-\tilde{i}_{d}^{e} \\
0 & \sigma & -\sigma
\end{array}\right]
$$

Then, the characteristic equation of Eq. (6) can be expressed as:

$$
\begin{aligned}
D(\bar{\lambda})= & |\bar{\lambda} E-J|=\bar{\lambda}^{3}+(2+\sigma) \bar{\lambda}^{2} \\
& +\left[1+2 \sigma+\sigma\left(\tilde{i}_{d}^{e}-\gamma\right)+\left(\tilde{\omega}_{g}^{e}\right)^{2}\right] \bar{\lambda} \\
& +\sigma\left[1+\tilde{i}_{q}^{e} \tilde{\omega}_{g}^{e}+\tilde{i}_{d}^{e}-\gamma+\left(\tilde{\omega}_{g}^{e}\right)^{2}\right]
\end{aligned}
$$

By studying the characteristic equation $D(\bar{\lambda})$, it is possible to judge if the equilibrium point of system (4) is stable in the sense of Lyapunov, and then determine the conditions for its Hopf bifurcation. Then, the mathematical conditions for the DPMSG to evolve from the limit cycle to Hopf bifurcation and produce chaotic motions can be identified theoretically.

Case 1: $\tilde{u}_{q}=\tilde{u}_{d}=\tilde{T}_{L}=0$

Here, $\tilde{u}_{q}=\tilde{u}_{d}=\tilde{T}_{L}=0$ means the generator has no input voltage or load. Under this assumption, the author explored the transient process of the D-PMSG operation after stopping power generation. If $\gamma>1$, the three equilibrium points of the system can be obtained as $\mathrm{S}_{0}(0,0,0)$ and $\mathrm{S}_{1,2}(\gamma-$ $1, \pm \sqrt{\gamma-1}, \pm \sqrt{\gamma-1}$ ) by solving Eq. (5). Among them, $\mathrm{S}_{0}$ is the zero-solution equilibrium point, while $S_{1,2}$ are nontrivial equilibrium points. For $\mathrm{S}_{0}$, the characteristic polynomial corresponding to Eq. (7) can be expressed as: 


$$
\begin{aligned}
& D(\bar{\lambda})=\bar{\lambda}^{3}+(2+\sigma) \bar{\lambda}^{2}+(1+2 \sigma-\sigma \gamma) \bar{\lambda}+\sigma(1-\gamma) \\
& =(\bar{\lambda}+1)\left[\bar{\lambda}^{2}+(\sigma+1) \bar{\lambda}+\sigma(1-\gamma)\right]
\end{aligned}
$$

Then, the eigenvalue corresponding to $\mathrm{S}_{0}$ can be obtained as $\bar{\lambda}_{1}=-1, \bar{\lambda}_{2,3}=\frac{-(\sigma+1) \pm \sqrt{(\sigma+1)^{2}-4 \sigma(1-\gamma)}}{2}$, where eigenvalue $\bar{\lambda}_{2}$ is a positive root. In this case, $\mathrm{S}_{0}$ is an unstable equilibrium point (saddle point). For $\mathrm{S}_{1,2}$, the polynomial of the corresponding Jacobian matrix can be expressed as:

$$
D(\bar{\lambda})=\bar{\lambda}^{3}+(2+\sigma) \bar{\lambda}^{2}+(\sigma+\gamma) \bar{\lambda}+2 \sigma(\gamma-1)
$$

According to Eq. (9), if $\gamma=\gamma_{h}=\frac{\sigma(\sigma+4)}{\sigma-2}(\sigma>2)$, the eigenvalue corresponding to $\mathrm{S}_{1,2}$ can be expressed as:

$$
\bar{\lambda}_{1}=-(\sigma+2), \bar{\lambda}_{2,3}= \pm j \sqrt{\frac{2 \sigma(\sigma+1)}{\sigma-2}}
$$

The following can be proved by advanced mathematical knowledge: If $\gamma<\gamma_{h}$, then $\operatorname{Re}\left(\bar{\lambda}_{2,3}\right)<0$; if $\gamma=\gamma_{h}$, then $\operatorname{Re}\left(\bar{\lambda}_{2,3}\right)=0$; if $\gamma>\gamma_{h}$, then $\operatorname{Re}\left(\bar{\lambda}_{2,3}\right)>0$. Therefore, the relationship of characteristics Eq. (7) can be obtained as: if $\gamma<\gamma_{h}, \mathrm{~S}_{0}$ is an unstable saddle point, while $\mathrm{S}_{1,2}$ are stable foci; if $\gamma=\gamma_{h}$, system (4) is in the critical state of Hopf bifurcation, showing a limit cycle; if $\gamma>\gamma_{h}, \mathrm{~S}_{1,2}$ are unstable foci and the system has chaotic solution.

If $\gamma<1$, the system has only one equilibrium point $\mathrm{S}_{0}(0$, $0,0)$ according to Eq. (5). In this case, the real part of the eigenvalue obtained by Eq. (7) $\operatorname{Re}\left(\lambda_{1,2,3}\right)<0$. Hence, the equilibrium point is stable, and the system is in the equilibrium state.

According to the above mathematical model, the coefficients $\sigma$ and $\gamma$ only depend on the parameters of the PMSG, making it difficult to satisfy $\gamma \geq \gamma_{h}$. Hence, it is concluded that: when the system parameters are constant, the system will not face Hpof bifurcation and will lose stability.

Case 2: $\tilde{u}_{d} \neq \tilde{u}_{q} \neq \tilde{T}_{L} \neq 0$

In this case, the PMSG operates under general conditions. The three solutions to $\widetilde{\omega}_{g}^{e}$ in the third equation of (5) can be obtained by the common way to solve cubic equations [23]:

$$
\begin{aligned}
& \tilde{\omega}_{g 1}^{e}=\sqrt{-\frac{q}{2}+\sqrt{\Delta}}+\sqrt{-\frac{q}{2}-\sqrt{\Delta}} \\
& \tilde{\omega}_{g 2}^{e}=\xi \sqrt{-\frac{q}{2}+\sqrt{\Delta}}+\xi^{2} \sqrt{-\frac{q}{2}-\sqrt{\Delta}} \\
& \tilde{\omega}_{g 3}^{e}=\xi^{2} \sqrt{-\frac{q}{2}+\sqrt{\Delta}}+\xi \sqrt{-\frac{q}{2}-\sqrt{\Delta}}
\end{aligned}
$$

where, $\xi=\frac{-1+\sqrt{3} j}{2} ; \Delta=\left(\frac{q}{2}\right)^{2}+\left(\frac{p}{3}\right)^{3} ; q=2\left(\tilde{T}_{L} / \sigma\right)^{3} / 27-$ $\left[\left(\widetilde{T}_{L} / \sigma\right)\left(\widetilde{U}_{d}-\gamma+1\right)\right] / 3+\left(\widetilde{T}_{L} / \sigma\right)-\widetilde{U}_{q}, p=\widetilde{U}_{d}-\gamma+1-$ $\left(\tilde{T}_{L} / \sigma\right)^{3} / 3$.

Substituting the above equation into the equilibrium state Eq. (5), the solutions to the other two state variables $\tilde{\imath}_{d}^{e}$ and $\tilde{\imath}_{q}^{e}$ in the equilibrium state can be obtained.

When $\Delta>0$, the equation has a real root and a pair of conjugate imaginary roots. Then, the currents $\tilde{l}_{d}^{e}$ and $\tilde{l}_{q}^{e}$ have imaginary roots, which makes no sense.
When $\Delta=0$, there are three real roots; if $p=q=0$, the three roots are all zeros, i.e. there exists an equilibrium point $S_{0}=\left(\tilde{u}_{d}, \frac{\tilde{T}_{L}}{\sigma}, 0\right)$; if $p, q \neq 0$, two of the three real roots are equal, i.e. there are two equilibrium points.

When $\Delta<0$, there are three unequal equilibrium points.

Therefore, it is only meaningful to consider the situation when $\Delta \leq 0$.

As analyzed above, the conditions for generating a pair of conjugate imaginary roots can be derived from the polynomial $D(\bar{\lambda})$ of the Jacobian matrix corresponding to each solution:

$$
\sigma \tilde{i}_{q}^{e} \tilde{\omega}_{g}^{e}=2+4 \sigma+\left(\sigma^{2}+\sigma\right)\left(\tilde{i}_{d}^{e}-\gamma\right)+2\left(\tilde{\omega}_{g}^{e}\right)^{2}+2 \sigma^{2}
$$

Eq. (12) is valid under three conditions: (1) the three inputs $\left(\tilde{u}_{d}, \tilde{u}_{q}, \widetilde{T}_{L}\right)$ are adjusted properly; (2) the system parameters are changed; (3) the wind speed varies. In any of the three conditions, the D-PMSG will witness a limit cycle and enter the chaotic state.

To further verify the chaotic motions of the D-PMSG, the maximum Lyapunov exponent (LE) can be computed in system simulation by $[24,25]$ :

$$
L E=\frac{1}{t_{N}-t_{0}} \sum_{j=1}^{N} \ln \left(\frac{d_{j}}{d_{0}}\right)
$$

where, $d_{1} \cdots d_{N}$ are calculated by the process in Figure 1. As shown in Figure 1, another trajectory is selected near the state trajectory $x(t)$; the distance at $t_{0}$ is denoted as $d_{0}$, and the distance after $\Delta t$ is denoted as $d_{1}$. During the simulation, the number of iterations $N$ was set to 1,000 and $\Delta t=1 \mathrm{~ms}$.

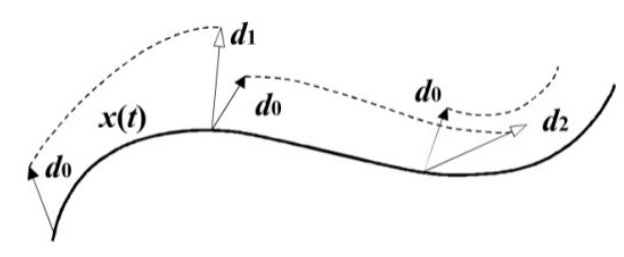

Figure 1. Calculation of the maximum LE

\section{ANALYSIS OF THE CHAOTIC FEATURES OF THE D-PMSG}

The actual parameters of the D-PMSG are as follows: the rated power $\mathrm{P}_{\mathrm{N}}=2.1 \mathrm{KW}$, rated voltage $\mathrm{U}_{\mathrm{N}}=220 \mathrm{~V}$, rated frequency $f=50 \mathrm{~Hz}$, sweep area of wind wheel $A=4 \mathrm{~m}^{2}$, air density $\rho=1.225 \mathrm{~kg} / \mathrm{m}^{3}$, number of pole pairs $n_{p}=17$, equivalent moment of inertia $J_{\text {eq }} \approx 0.0013 \mathrm{~kg} \cdot \mathrm{m}^{2}, L=$ $2.7 \mathrm{mH}$, resistance of stator $R_{S}=1.14 \Omega$, flux linkage of the permanent magnet $\psi_{f}=212 \mathrm{mH}$, rotational viscosity of the generator $B_{m}=8.65 \mathrm{~N} \cdot \mathrm{m} \cdot(\mathrm{rad} / \mathrm{s})^{-1}$, optimal tip-speed ratio $\lambda_{\text {opt }}=0.9$, and maximum wind power utilization factor $C_{p \max }$. According to these parameters, it can be obtained that $\sigma=16$ and $\gamma=45.92$.

Case 1: $\tilde{u}_{q}=\tilde{u}_{d}=\tilde{T}_{L}=0$

As shown in Figure 2(a), if $\gamma<1$, the system always tended to the equilibrium point $(0,0,0)$, whichever the initial 
condition, indicating that the system motions are stable under this condition. As shown in Figure 2(b), if $\gamma<\gamma_{h}$, there were two stable foci in the system, meaning that the system is table. As shown in Figure 2(c), if $\gamma \approx \gamma_{h}$, the limit cycle appeared in system operation, revealing that the system is in the critical state of Hopt bifurcation and that the stability of the equilibrium points is about to change. As shown in Figure 2(d), if $\gamma>\gamma_{h}$, none of the three equilibrium points were stable, showing that the system has entered the chaotic state.

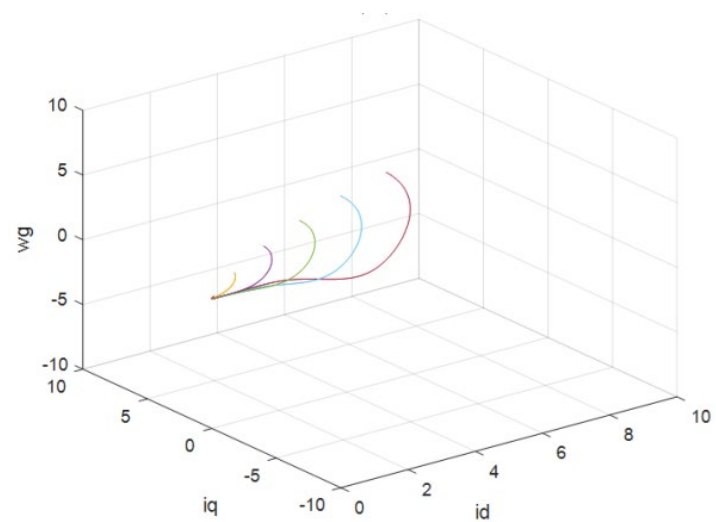

(a) $\gamma<1$

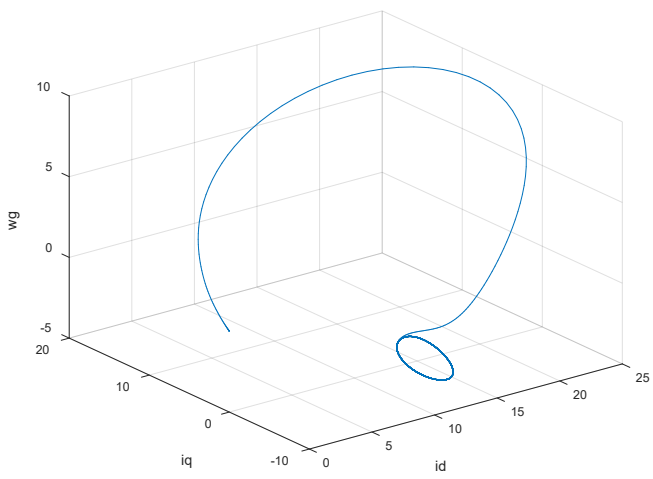

(c) $\gamma \approx \gamma_{h}$

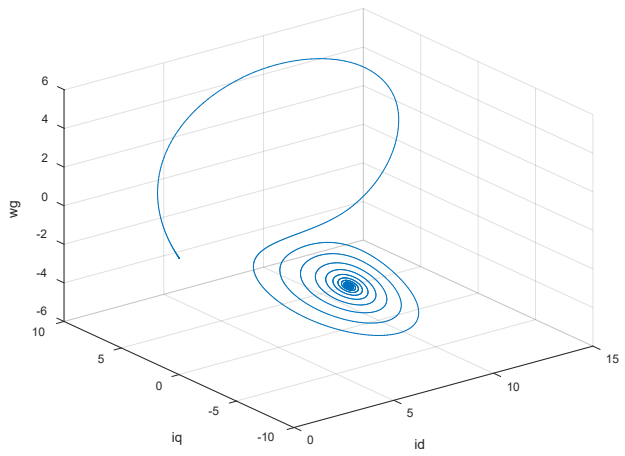

(b) $\gamma=9.85$

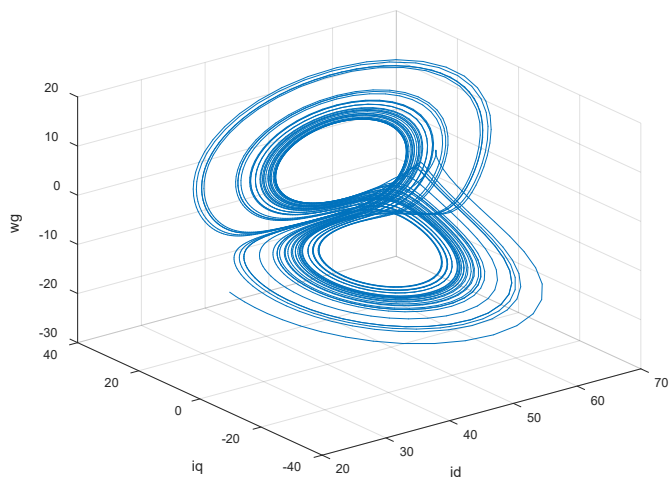

(d) $\gamma=45.92$

Figure 2. Three-phase diagram of system state variables

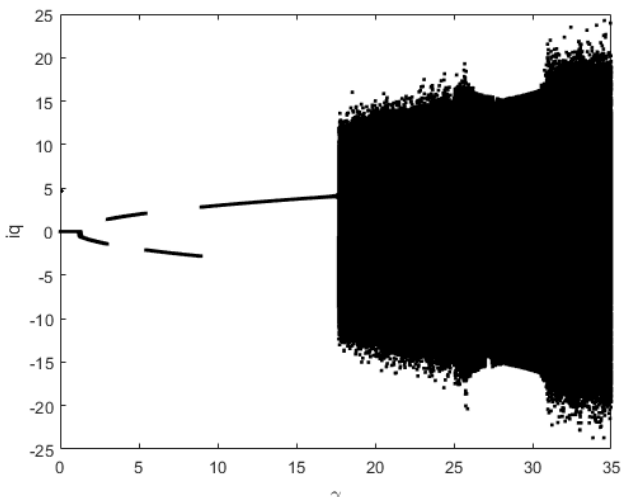

(a) Bifurcation map

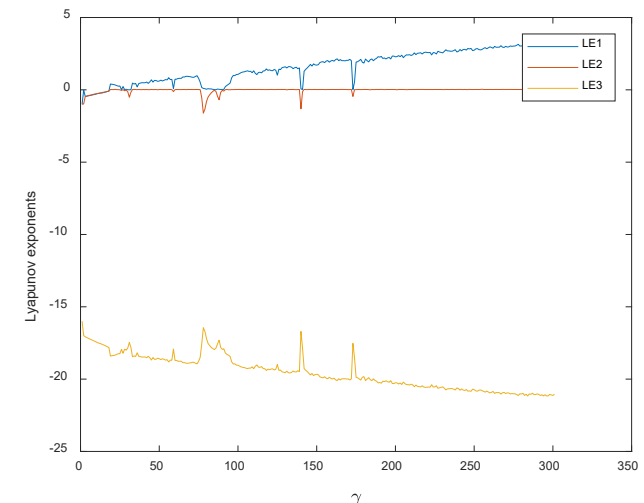

(b) LE spectrum

Figure 3. Bifurcation map and LE spectrum of the system

Figure 3(a) is the bifurcation diagram of the system under the initial conditions of $(20,0.1,-5)$, with $\gamma$ as the bifurcation parameter and $i_{q}$ as the state variable. It can be seen that, when $\gamma<1$, the system had only one stable equilibrium point; when $1<\gamma<\gamma_{h}$, there were two stable foci in the system, and the system stability depends on the initial value; when $\gamma>\gamma_{h}$, the system's equilibrium points mutated, and the system entered the chaotic state. As shown in Figure 3(b), when $\gamma=18.83$, the three LEs were $(0.15,0$, -
18.13), i.e. the system had positive LEs; in this case, bifurcation appeared and the system belonged to the chaotic state. When $\gamma=86$, the three LEs were $(0,-0.11,-17.9)$, and the system returned to regular motions, but ended up in chaotic state.

Case 2: $\tilde{u}_{d} \neq \tilde{u}_{q} \neq \tilde{T}_{L} \neq 0$

Figure 4(a) presents the three-phase diagram at $\tilde{u}_{d}=$ $-0.542, \tilde{u}_{q}=0.824$ and the wind speed of $v=7.6 \mathrm{~m} / \mathrm{s}$. It 
can be seen that the motion trajectory of the system converged to the stable equilibrium point slowly, but the system steadily entered the stable operation state after a period of time. When the wind speed gradually increased to $v=10.2 \mathrm{~m} / \mathrm{s}$, the limit cycle appeared (Figure 4(b)), and the system belonged to the critical stability state. When the wind speed further rose to $v=$ $13 \mathrm{~m} / \mathrm{s}$, the motion trajectory of the system is shown in Figure 4(c). Obviously, the three equilibrium states were all unstable,

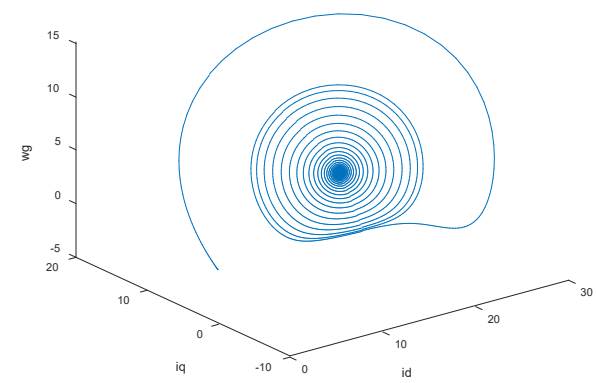

(a) Three-phase diagram of the system at $v=7.6 \mathrm{~m} / \mathrm{s}$ and the chaotic attractor appeared, indicating that the system entered the chaotic state. Hence, if $\tilde{u}_{d}, \tilde{u}_{q}$ and system parameters are constant, the variation in wind speed will cause changes to the torque of the turbine $T_{r}$. Then, the $\widetilde{T}_{r}$ obtained through affine transform and timescale transform also changes. As a result, the system moves from the equilibrium state to chaotic state.

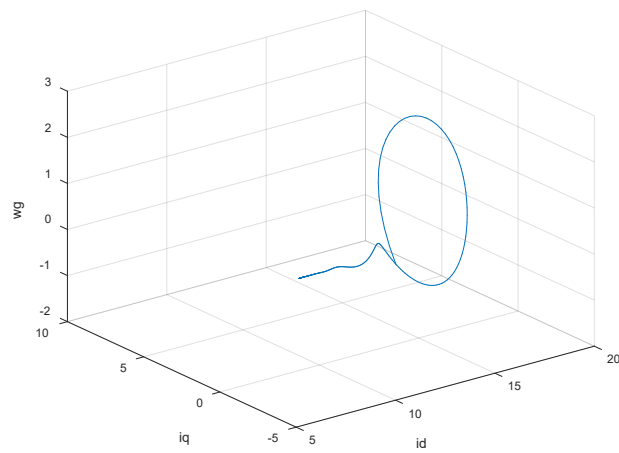

(b) Three-phase diagram of the system at $v=10.2 \mathrm{~m} / \mathrm{s}$

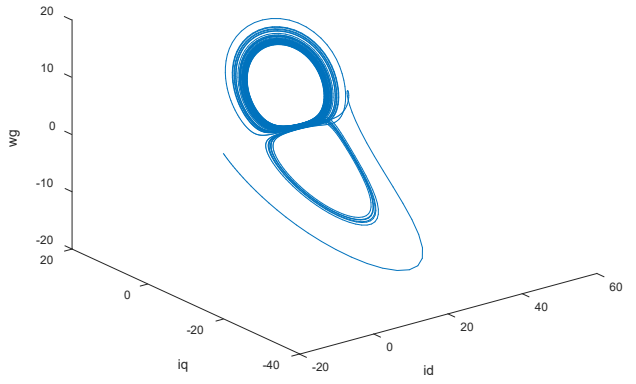

(c) Three-phase diagram of the system at $v=13 \mathrm{~m} / \mathrm{s}$

Figure 4. Three-phase diagrams of the system at different wind speeds

Figure 5 provides the three-phase diagrams with wind speed $v$ as the bifurcation parameter and $i_{q}$ as the state variable. As shown in Figure 5, when $v \leq 10.5 \mathrm{~m} / \mathrm{s}$, the system operated in a stable manner; when the wind speed increased to $v=$ $10.5 \mathrm{~m} / \mathrm{s}$, the bifurcation occurred and the system entered the chaotic state.

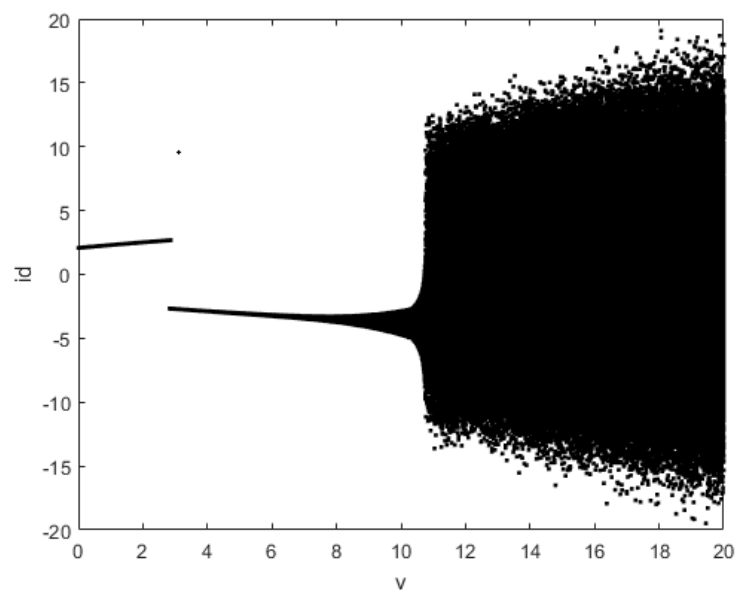

Figure 5. Bifurcation map of the system at different wind speeds $\mathrm{V}$

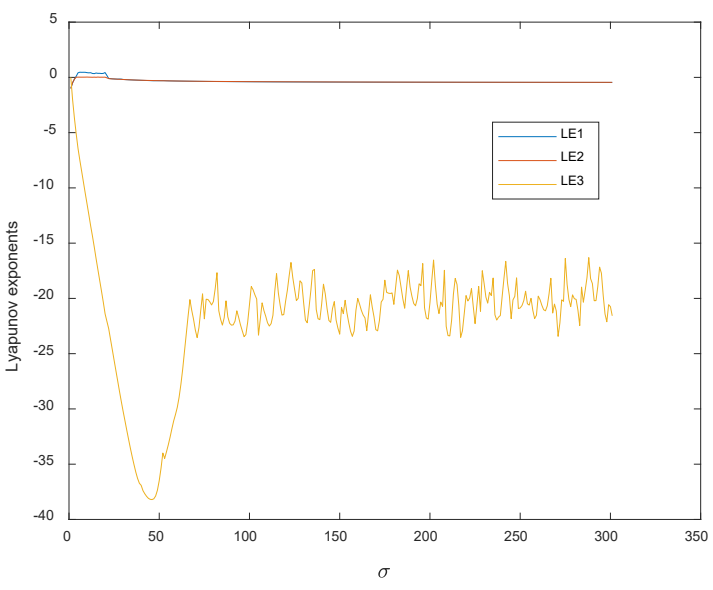

Figure 6. The LE spectrum of the system with $\sigma$ as the variable

Figure 6 shows the LE spectrum with $\sigma$ as the variable. It can be seen that, if $\sigma \in[4.1,13.6)$, the three LEs are LE1 $>0$, $\mathrm{LE} 2=0$, and $\mathrm{LE} 3<0$, i.e. the system had positive LEs; in this case, bifurcation appeared and the system belonged to the chaotic state. Therefore, the system becomes chaotic, if the parameter $\sigma$ changes or falls in a certain interval. This finding 
echoes with the theoretical result in the preceding section. In other words, the simulation results agree with the theoretical derivation. For a D-PMSG, the system will lose stability facing changes in system parameters or wind speed. This conclusion has practical significance in actual engineering.

\section{CONCLUSIONS}

This paper drives the chaotic model of the D-PMSG through affine transform and timescale transform. In addition, the Lyapunov stability theory was introduced to analyze the parameter conditions for the D-PMSG to move from equilibrium state, the limit cycle state to the chaotic state under different inputs. The analysis proves the existence of chaotic attractor under certain conditions. In addition, the chaotic features of the D-PMSG were simulated, using three-phase diagram, bifurcation diagram and the LE spectrum. The simulation results show that the chaotic attractor will appear in the D-PMSG, when there are changes to system parameters or the wind speed, which severe affects the stable operation of the D-PMSG. For actual wind power systems, the stable operation conditions, parameter uncertainty and wind speed disturbance are even more complex. Therefore, the future research will probe deeper into the chaotic motions and chaotic control of the D-PMSG.

\section{ACKNOWLEDGMENTS}

This work has been supported by the National Natural Science Foundation of China (51907128) and Deyang Science and Technology Plan Project(2019SZ80).

\section{REFERENCES}

[1] Karik, F., Sozen, A., Izgec, M.M. (2017). The importance of wind power forecasts: A case study in Turkish electricity market. Journal of PolytechnicPoliteknik Dergisi, 20(4): 851-861. https://doi.org/10.2339/poloteknik.369038

[2] Barutha, P., Nahvi, A., Cai, B., Jeong, H.D., Sritharan, S. (2019). Evaluating commercial feasibility of a new tall wind tower design concept using a stochastic levelized cost of energy model. Journal of Cleaner Production, 240: 118001. https://doi.org/10.1016/j.jclepro.2019.118001

[3] Elsonbaty, N.A., Enany, M.A., Diab, A.M. (2018). Proposed control policy for high power transfer capability DFIC. Electric Power System Research, 155: 144-152. https://doi.org/10.1016/j.epsr.2017.10.012

[4] Kang, T., Kim, M., Lee, S.Y., Kim, Y.C. (2017). Modeling and a simple multiple adaptive control of PMSM drive system. Journal of Power Electronics, 17(2): 442-452. https://doi.org/10.6113/JPE.2017.17.2.442

[5] Kondo, K., Doki, S. (2017). Position estimation system for PMSM position sensorless control in inverter overmodulation drive. IEEJ Journal of Industry Applications, 6(3): 165-172. https://doi.org/10.1541/ieejjia.6.165

[6] Rezaei, N., Mehran, K., Cossar, C. (2019). A modelbased implementation of an MPPT technique and a control system for a variable speed wind turbine PMSG. International Journal of Modelling, Identification and Control,

31(1):

$3-15$.
https://doi.org/10.1504/IJMIC.2019.096822

[7] Abdelrahem, M., Hackl, C.M., Kennel, R. (2018). Finite position set-phase locked loop for sensorless control of direct-driven permanent-magnet synchronous generators. IEEE Transactions on Power Electronics, 33(4): 30973105. https://doi.org/10.1109/TPEL.2017.2705245

[8] Yang, X.H., Xu, S.P., Liu, H.S., Liu, G.P. (2012). Chaos analysis and extended adaptive chaos control of permanent magnet synchronous motors. Journal of Convergence Information Technology, 7(18): 58-65. https://doi.org/10.4156/jcit.vol7.issuel8.7

[9] He, R., Han, Q. (2017). Dynamics and Stability of Permanent-Magent Synchronous Motor. Mathematical Problems in Engineering. https://doi.org/10.1155/2017/4923987

[10] Hemati N. (1994). Strange attractors in brushless DC motors. IEEE Transaction on Circuits and Systems I: Fundamental Theory and Applications, 41(1): 40-45. https://doi.org/10.1109/81.260218

[11] Li, Z., Park, J.B., Joo, Y.H., Zhang, B., Chen, G.R. (2002). Bifurcations and chaos in a permanent-magnet synchronous motor. IEEE Transactions on Circuits and Systems I: Fundamental Theory and Applications, 49(3): 383-387. https://doi.org/10.1109/81.989176

[12] Chen, X., Hu, J.B., Peng, Z.X., Yuan, C.H. (2017). Bifurcation and chaos analysis of torsional vibration in a PMSM based driven system considering electromechanically couple effect. Nonlinear Dynamics, 88(1): 277-292. Https://doi.org/10.1007/s11071-0173419-z

[13] Xue, W., Li, Y.L., Cang, S.J., Jia, H.Y., Wang, Z.H. (2015). Chaotic behavior and circuit implementation of a fractional-order permanent magnet synchronous motor. Journal of the Franklin Institute, 352(7): 2887-2898. https://doi.org/10.1016/j.jfranklin.2015.05.025

[14] Chang, X., Liu, L., Ding, W., Liang, D., Liu, C., Wang, H., Zhao, X. (2017). Novel nonsingular fast terminal sliding mode control for a PMSM chaotic system with extended state observer and tracking differentiator. Journal of Vibration and Control, 23(15): 2478-2493.

[15] Yang, G.L., Li, H.G. (2009). Sliding mode variablestructure control of chaos in direct-driven permanent magnet synchronous generators for wind turbines. Acta Physica Sinica, 58(11): 7552-7557.

[16] Wang, L., Li, Y.H., Lu, G.L., Zhu, X.H. (2011). Tracking controller of D-PMSG chaos motion. Electric Power Automation Equipment, 31(6): 45-49.

[17] Chen, J.H., Yau, H.T., Hung, W. (2014). Design and study on sliding mode extremum seeking control of the chaos embedded particle swarm optimization for maximum power point tracking in wind power systems. Energies, $\quad 7(3)$ : 1706-1720. https://doi.org/10.3390/en7031706

[18] Cao, N., Shi, W.X., Zhu, C.H. (2017). Simulated analysis of dynamic characteristics of permanent magnet directdrive synchronous wind turbine generators. Electric Machines \& Control Application, 44(1): 104-109.

[19] Si, G., Zhu, J., Diao, L., Ding, Z. (2017). Modeling, nonlinear dynamic analysis and control of fractional PMSM of wind turbine. Nonlinear Dynamics, 88(2): 985-1000. https://doi.org/10.1007/s1 1071-016-3289-9

[20] Barros, L.S., Barros, C.M.V. (2017). An internal model control for enhanced grid connection of direct-driven PMSG based wind generators. Electric Power Systems 
Research,

151:

440-450.

https://doi.org/10.1016/j.epsr.2017.06.014

[21] Boubellouta, A., Boulkroune, A. (2019). Chaos synchronization of two different PMSMs via a fractionalorder sliding mode controller. International Journal of Researcher, 60(2): 165-174. https://doi.org/10.1504/IJCAT.2019.100135

[22] Yu, X.H. (1996). Controlling Lorenz chaos. International Journal of Systems Science, 27(4): 355-359. https://doi.org/10.1080/00207729608929224

[23] Luo, W., Cui, X.S., Luo, Y.L. (2018). Approximate solutions of a class of cubic characteristic equations in one variable in asymmetric transient analysis of induction machines. Proceedings of the CSEE, 28(27): 126-130.

8013.pcsee.2008.27.016

[24] Hun, Z.Y., Zhou, Y.C. (2018). One-dimensional nonlinear model for producing chaos. IEEE Transactions on Circuits and Systems I: Regular Papers. 65(1): 235246. https://doi.org/10.1109/TCSI.2017.2717943

[25] Mobayen, S., Kingni, S.T., Pham, V., Nazarimehr, F., Jafari, S. (2018). Analysis, synchronization and circuit design of a new highly nonlinear chaotic system. International Journal of Systems Science, 49(3): 617-630. https://doi.org/10.1080/00207721.2017.1410251 